\section{Orbital blow-out fractures: surgical timing and technique}

GJ Harris

\begin{abstract}
Purpose To recommend a tailored approach to surgical timing in the repair of orbital blow-out fractures, and to offer suggestions for improved functional and aesthetic surgical outcomes.

Methods Traditional guidelines for surgical timing are reviewed. An evidence-based approach that considers soft-tissue disruption relative to bone-fragment separation is presented. The author's techniques for repair of isolated orbital floor, isolated medial wall, and combined floor-medial wall fractures are presented.
\end{abstract}

Results As demonstrated previously, greater degrees of soft-tissue incarceration or displacement, with presumably greater intrinsic damage and subsequent fibrosis, result in poorer motility outcomes despite complete release of soft tissues. There is a suggestion that earlier intervention for such injuries might improve outcomes. Lower fornix and transcaruncular incisions, careful extrication of incarcerated tissue, and thin alloplastic implants have proven successful in the author's hands.

Conclusions The degree of soft-tissue displacement relative to bone fragment distraction, as depicted in preoperative computed tomography (CT) scans, should be considered in the timing of surgery. Incisions, soft-tissue handling, and implant material, thickness, and positioning can all affect the functional and aesthetic outcomes.

Eye (2006) 20, 1207-1212. doi:10.1038/sj.eye.6702384

Keywords: orbit; blow-out; fracture

\section{Introduction}

Among facial fractures, orbital blow-out injuries most often involve ophthalmologists in their care. The election and timing of surgical repair are areas of continued interest and some controversy. If timing is significant, then the physician who first evaluates the patient - even if he or she does not perform the surgery - may influence the clinical outcome by the timing of referral.

\section{Surgical timing}

The term 'blow-out fracture' was coined in 1957, when Smith and Regan ${ }^{1}$ described the mechanism of injury. They produced an impact on the orbital soft tissues of a cadaver, increasing hydraulic pressure, and causing the thin, internal walls to fracture. Soft tissues were displaced or incarcerated, correlating with enophthalmos and restricted motility.

For the next two decades, when diagnosis was based on plain X-rays and hypocycloidal tomography, treatment recommendations ranged from early intervention for all fractures to prolonged observation in all cases, with delayed surgery reserved for late enophthalmos or persistent diplopia. ${ }^{2,3}$

The advent of computed tomography (CT) narrowed the poles of the debate, and produced a fairly uniform protocol in the 1980s and 1990s. ${ }^{4}$ This approach continues to be used in many centers. If the fracture is large, enophthalmos is anticipated and surgery is usually performed within the first 2 weeks. Enophthalmos may be obvious at the time of presentation, or it may be masked by oedema or haematoma. If surgery is delayed until enophthalmos is apparent, then fibrosis between orbital soft tissues, sinus mucosa, and bone fragments can make surgery more difficult.

In this paradigm, a 'small' fracture refers to a minimally displaced fracture. In fact, some trap-door injuries may appear so well aligned that no fracture is identified by the radiologist (Figure 1). However, at the time of the injury,
Section of Orbital and Ophthalmic Plastic Surgery, Department of Ophthalmology, Medical College of Wisconsin, Milwaukee, WI, USA

Correspondence: GJ Harris, Eye Institute, 925 North 87th Street, Milwaukee, WI, 53226, USA

Tel: + 1414456 7989;

Fax: + 14144566300

E-mail: gjharris@

mcw.edu

Received: 12 March 2006 Accepted: 22 March 2006

The author has no financial or proprietary interest related to this article 


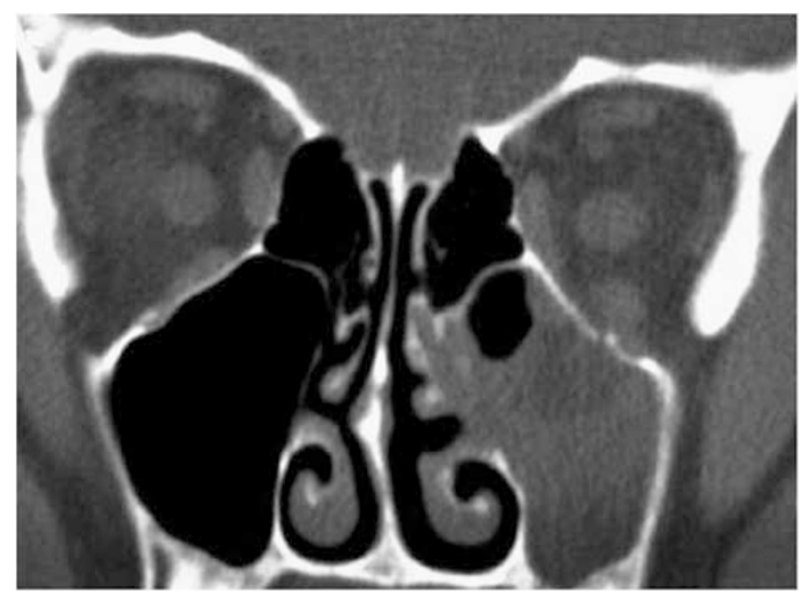

Figure 1 A well-aligned left orbital trap-door fracture in a patient with significant globe restriction. Note the different radiodensities of orbital fat and blood within the maxillary sinus. Rounding of the muscle contour, compared to the normal side, suggests that the contiguous fibrofatty tissue is incarcerated.

there was significant transient displacement of bone fragments, which captured soft tissue when they snapped back into position. In these cases, motility, rather than volume, is the primary concern, and surgery is usually advised only if there is clinically significant diplopia that does not resolve within 2 weeks.

In the last two decades, orbital volume outcomes were generally well served using this approach to blow-out fractures. However, motility outcomes were less than ideal. In an extreme example, Emery et al ${ }^{5}$ described 49 patients; although 43 had surgery within 2 weeks of the injury, almost $50 \%$ of the total had diplopia 6 or more months following surgery. These outcomes prompted our group to look for preoperative criteria that might predict the postoperative course, and perhaps influence initial therapy.

More than 25 years ago, Leo Koornneef ${ }^{6}$ demonstrated a complex network of fibrous septa that functionally unite the sheath of the inferior rectus muscle, the fibrofatty tissues, and the periosteum of the orbital floor. In orbital fractures, motility may be limited by displacement of this entire complex, or by entrapment of any of its components. (The muscle, per se, need not be lodged between bone fragments.) However, if displacement and entrapment were the only determinants of final motility, then surgical reduction of soft tissues should be curative-but outcome studies suggest otherwise.

We reasoned that the soft tissues, when driven between bone fragments, are damaged, and they may be further damaged after bone fragments have repositioned themselves. That ongoing tissue injury leads to fibrosis, which can tether the globe, despite complete surgical release. We wondered if the extent of that damage might predict the postoperative motility.
We reviewed a series of 30 patients with blow-out fractures, who had retrievable CT scans, preoperative diplopia, a uniform surgical procedure by the same surgeon $(\mathrm{GJH})$ - including complete release of entrapped tissue - and binocular visual fields at least 1-3 months after surgery. ${ }^{7}$ Motility outcomes were quantified by one group of our investigators, who were unaware of the CT or surgical findings. They measured the vertical fusion within binocular visual fields (Figure 2).

Another group of investigators, unaware of the visual fields or surgical findings, analysed preoperative $\mathrm{CT}$ scans, and designated each fracture as either ' $\mathrm{A}$ ' or ' $\mathrm{B}$ ', judging - for any given configuration of bone fragments - whether the soft-tissue disruption was major or minor (Figure 3). ${ }^{7}$ These investigators evaluated the entire coronal series and based their decision on the sections with the greatest distortion. For example, among trap-door injuries, the milder designation was used if no orbital tissue extended into the maxillary sinus. We assumed greater damage if tissue had been driven between bone fragments into the sinus, and then was left compressed and ischaemic after the fragments had snapped back. If bone fragments were distracted or separated, the soft-tissue displacement was judged to be proportionate or disproportionate to the bone separation - with added compression or attenuation of the blood supply in the more severe cases. Finally, if bone fragments surrounded the soft-tissue complex, motility was limited not by entrapment, but by translocation of the entire functional unit. This could be mild, or severe with greater stretch of the soft tissues.

Clearly, the designation of fractures as either ' $\mathrm{A}$ ' or ' $\mathrm{B}$ ' is very subjective. Fractures represent a continuum, and any classification is artificial. Nevertheless, when examiners unfamiliar with the clinical findings evaluated the scans, their subjective sense of greater vs lesser soft-tissue distortion did correlate with postoperative motility. The 30 patients were ordered by degrees of vertical fusion at 4-10 weeks after surgery. With reference to the median outcome, half the patients had fusion of $86^{\circ}$ or less, and half had fusion of $88^{\circ}$ or more. Among those with outcomes poorer than the median, 'B' fractures predominated 3:1 (73 vs 27\%). Among those with better outcomes, ' $A$ ' fractures predominated 2:1 (67 vs $33 \%$ ). Moving away from the median, differences were even more defined. Among patients with less than $80^{\circ}$ of vertical fusion, $91 \%$ had ' $\mathrm{B}$ ' fractures. Among those with greater than $105^{\circ}, 78 \%$ had ' $\mathrm{A}$ ' fractures.

Although, as a group, the so-called ' $\mathrm{B}$ ' fractures had a poorer prognosis - by more than 2:1 (11 patients vs five patients) - the timing of surgery within the group is of interest. Among those with less than the median outcome, only one of 11 patients had surgery during the 


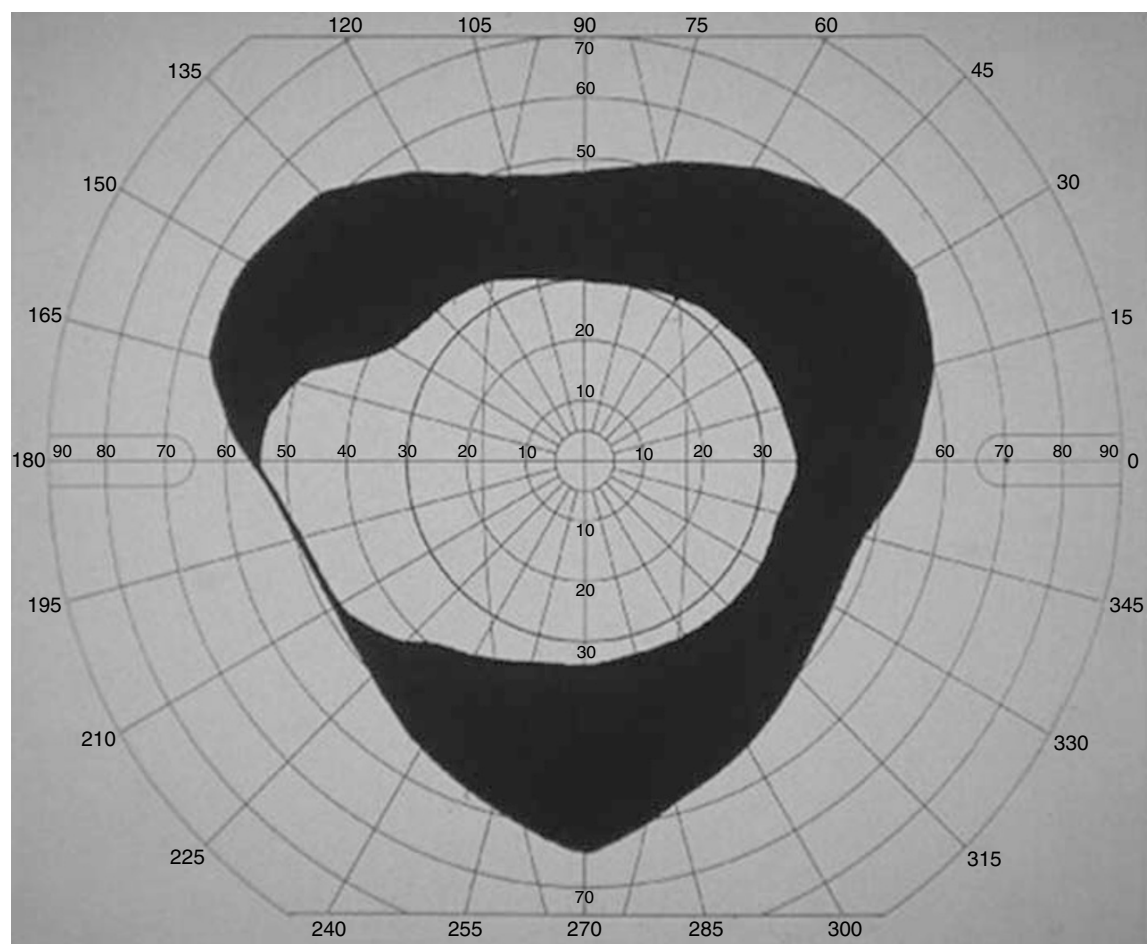

Figure 2 Field of single binocular vision 10 weeks after surgery. Vertical fusion measures $64^{\circ}$. Shaded area represents diplopia in this case and depicts the full normal binocular visual field according to the format of Feibel and Roper-Hall ${ }^{8}$ (reprinted with permission from Harris $e t a l^{7}$ ).

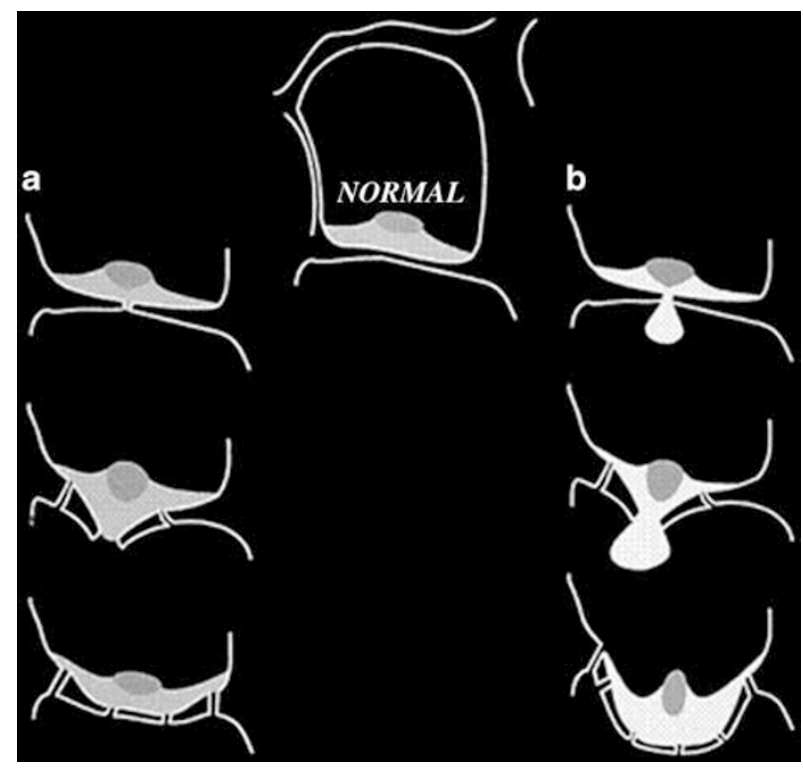

Figure 3 Schematic classification of blow-out fractures, based on lesser (a) or greater (b) soft-tissue distortion relative to bone fragment configuration (reprinted with permission from Harris et $\left.a l^{7}\right)$.

first week after injury. Among the five patients with better results, three had surgery during the first week. The raw numbers are very small, but they suggest that

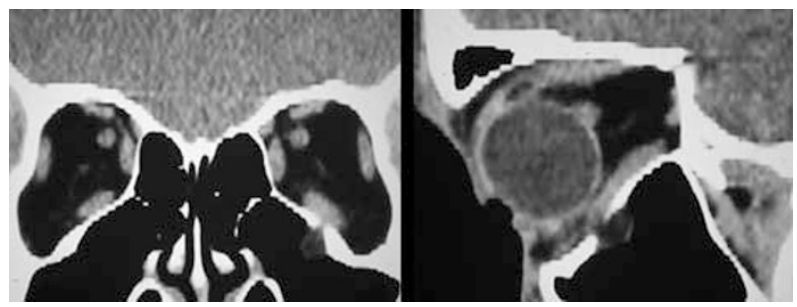

Figure 4 (Left) Coronal and (right) sagittal CT scans demonstrate soft-tissue displacement disproportionate to bone fragment separation.

this type of fracture might still have a favourable outcome if repaired promptly.

The initial contusion, shearing, and laceration cannot be undone, but early reversal of ongoing tissue crush or severe stretch might limit late fibrosis, and for fractures with disproportionate soft-tissue displacement (Figure 4), we aim for repair within 1-3 days of the injury. Surgery within this interval is not always logistically possible, but we certainly no longer wait 2 weeks to intervene. Others have also recommended early surgery for these trap-door injuries, which are most common in children. Jordan et al' termed them 'white-eyed blow-out fractures', because severely restricted movement can occur with otherwise quiet eyes. 


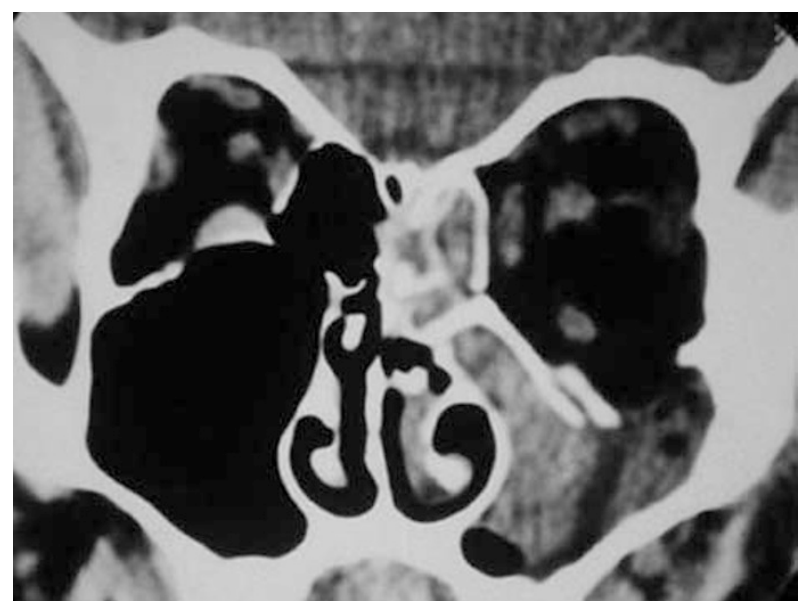

Figure 5 Soft-tissue and bone displacement are proportionate. Fracture size and displacement portend probable enophthalmos without treatment.

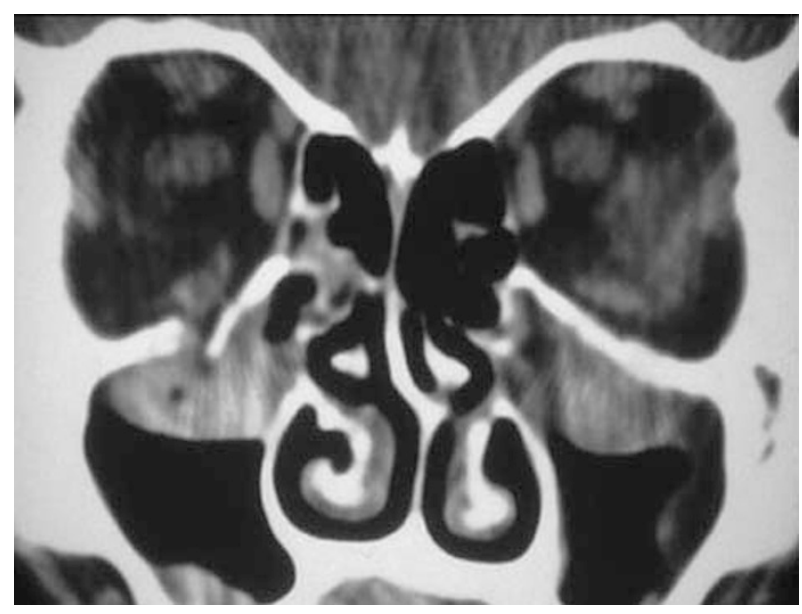

Figure 6 Fat extends through the fracture site, but soft-tissue and bone displacement are commensurate, allowing the tissue to stretch and restore full fusional amplitude. Surgery was not performed, and the patient's post-traumatic diplopia resolved spontaneously within 2 weeks of the injury (reprinted with permission from Harris $\mathrm{et}^{\mathrm{7}} \mathrm{l}^{\mathrm{T}}$ ).

Fractures with proportionate soft-tissue and bone displacement can be managed according to size. If enophthalmos is anticipated (Figure 5), surgery does need not be delayed, although it can be if necessary (eg, oedema, hyphema, or retinal detachment).

If late enophthalmos is not likely (Figure 6), patients can be observed for a full 2 weeks - or longer if improving. With substantial improvement, surgery may be totally avoided.

\section{Surgical technique}

For cases that do require surgery, there are some differences in perspective between ophthalmic and nonophthalmic surgeons who venture into the orbit.

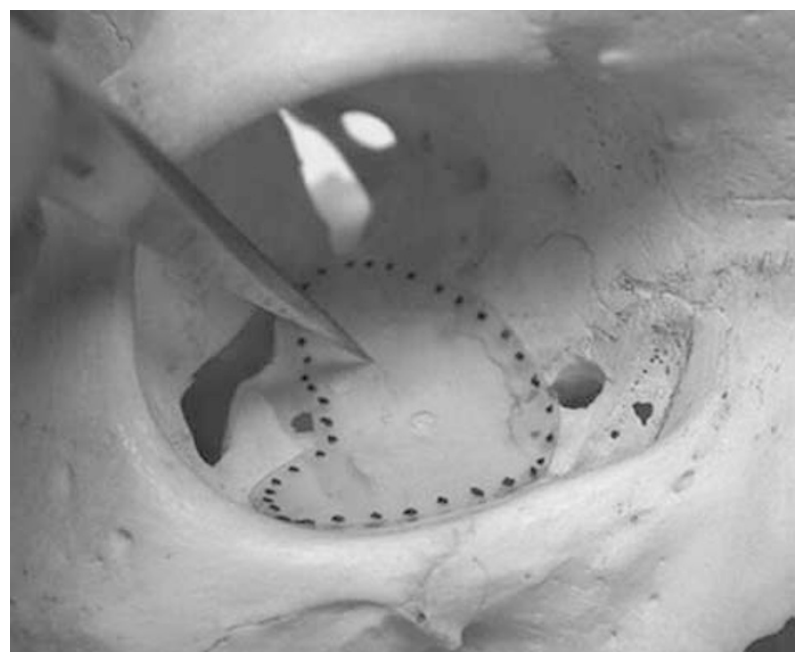

Figure 7 Nylon foil implant $(0.3 \mathrm{~mm}$ thickness $)$ should rest behind the orbital rim, bridge the infraorbital canal, and be supported by an intact bony ledge posteriorly.

The orbital floor can be accessed through a skin incision - subciliary, at a natural crease, or over the inferior orbital rim - or through the lower fornix. The last avoids a visible scar, and is less likely to result in lid retraction. It begins with a small lateral canthotomy and inferior cantholysis, which completely disinsert the lower lid. The orbital rim is then engaged between retractors. I incise the conjunctiva with scalpel or scissors, and incise the remaining tissues with a needle-tipped monopolar cautery, gaining access to the subperiosteal space. The limits of the fracture are then defined, and the entrapped tissue carefully released. Although other disciplines may see these injuries as bone problems, we view them as soft-tissue problems. Bone fragments may have to be removed to atraumatically release the herniated tissue.

With regard to an implant, some specialists favour cranial bone grafts. However, because about $50 \%$ resorption is anticipated, overcorrection with thick sheets of bone is intentionally performed. As a result, the globe may be superiorly displaced and motility may be impaired during the 6-12 months needed for resorption. I prefer thin alloplastic materials, and generally use a 0.3-mm-thick nylon foil (SupraFOIL ${ }^{\circledR}$, S Jackson Inc., Alexandria, VA, USA). This material will support the orbital soft tissues if it rests on intact bone peripheral to the defect, yet it will slightly bow under the weight of the tissues, and reproduce the natural contour of the floor. The implant is fashioned to rest behind the infraorbital rim anteriorly, and on an intact bony ledge posteriorly. It should bridge the infraorbital nerve, and not impinge on the lacrimal sac or inferior oblique muscle origin (Figure 7). Fixation may not be necessary, but I like to use fibrin glue, which will stabilize the implant until 
fibrosis develops around and through the implant's perforations.

Closure includes the periosteum with a 6-0 polyglactin suture, and the conjunctiva with 6-0 plain catgut. The lateral canthus is reconstituted with a 6-0 polyglactin

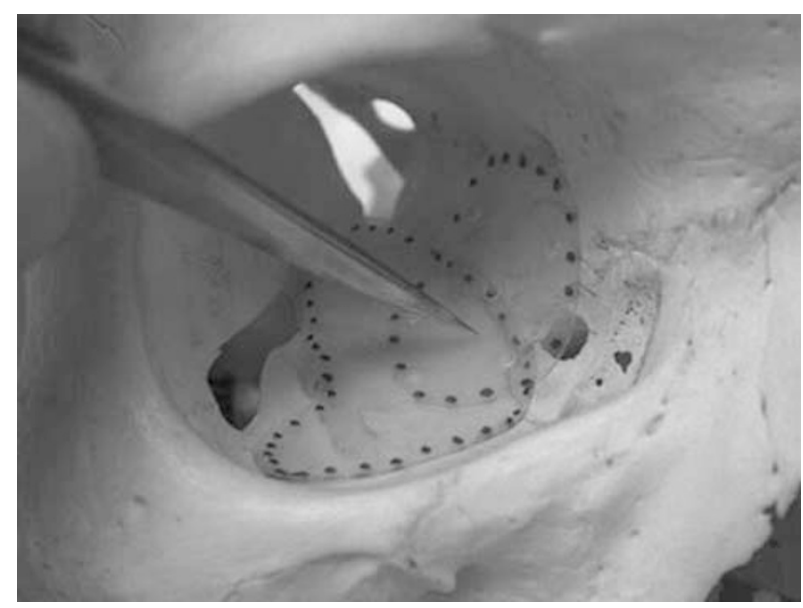

Figure 8 Desired positioning of floor and medial wall implants for combined fractures. Stability requires intact lateral floor and superior medial wall segments. suture, and the small cutaneous incision is closed with 6-0 plain catgut. I place a fluted drain into the subperiosteal space to avoid a haematoma that might compromise vision. It also reduces the amount of ecchymosis and oedema. This is removed on the first postoperative morning. Preplaced 7-0 nylon sutures within the drain site are then tied to reduce visible scarring.

Blow-out fractures may be limited to the medial wall. For many years, the approach to the medial wall involved a Lynch incision, which can leave an unsightly, webbed scar. A preferable alternative is the transcaruncular approach, popularized by the University of California-Los Angeles group for several indications. ${ }^{10}$ Fractures occur in the thin lamina papyracea inferior to the anterior and posterior ethmoidal foramina. Once the incarcerated tissue has been released, a thin nylon foil is inserted, and supported on stable bone peripheral to the fracture.

Combined floor and medial wall fractures are more complicated than either alone, because there may not be peripheral bony support for standard implants. Options include a metallic implant or mesh fixed to the stable orbital rim. The MEDPOR Channel implant ${ }^{\mathbb{R}}$ (Porex
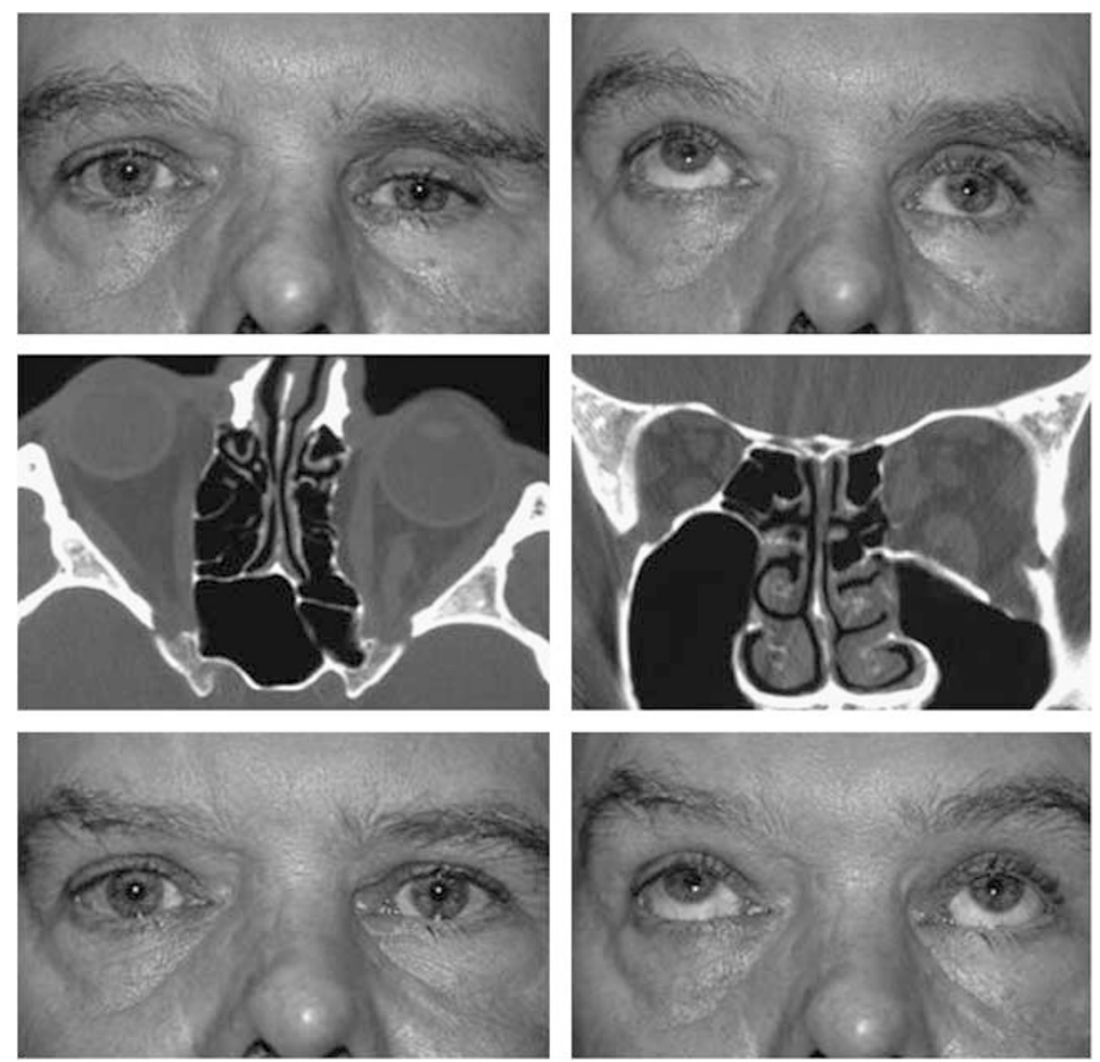

Figure 9 (Top, left, and right) Patient with severe left enophthalmos, inferior globe displacement, and diplopia 2 years after primary repair by another specialty service. (Centre, left, and right) note the depression and angulation at the floor-medial wall junction, compared with the normal contour of the opposite side. Repair involved combined floor and medial wall surgical approaches and two implants. (Bottom, left, and right) improved symmetry and function. 
Corporation, Fairburn, GA, USA) employs a similar principle, and is technically easier to use, because it does not have sharp edges that can snag soft tissue. Another option is a tailored floor-medial wall sheet that relies on anterior, lateral, posterior, and superior stable bone. Like the others, this requires somewhat awkward insertion through the lower fornix incision, around the inferior oblique muscle and lacrimal sac.

For the past few years, I have found that I can reduce the herniated tissues less traumatically if I use both lower fornix and transcaruncular approaches. Therefore, I place an implant through each incision. If one sheet extends into the breach, the other will rest on it, and will bow enough to simulate the normal contour. If both extend into the breach (ie, meet at a $90^{\circ}$ angle), a small third sheet can span the other two (Figures 8 and 9).

In summary, I suggest thinking of a blow-out fracture as a soft-tissue injury. Consider the degree of soft-tissue disruption - as depicted in CT scans - in the timing of surgery. Incisions, soft-tissue handling, and implant material, thickness, and positioning can all affect the functional and aesthetic outcomes.

\section{Acknowledgements}

This work is supported in part by core Grant EY01931 from the National Eye Institute, Bethesda, Maryland, and by an unrestricted grant from Research to Prevent Blindness, Inc., New York, NY, USA.

\section{References}

1 Smith B, Regan WF. Blow-out fracture of the orbit: mechanism and correction of internal orbital fracture. Am J Ophthalmol 1957; 44: 733-739.

2 Converse JM, Smith B, Obear MF, Wood-Smith D. Orbital blowout fractures: a ten-year survey. Plast Reconstr Surg 1967; 39: 20-36.

3 Putterman AM, Stevens T, Urist MJ. Nonsurgical management of blow-out fractures of the orbital floor. Am J Ophthalmol 1974; 77: 232-239.

4 Wilkins RB, Havins WE. Current treatment of blow-out fractures. Ophthalmology 1982; 89: 464-466.

5 Emery JM, von Noorden GK, Schlernitzauer DA. Orbital floor fractures: long-term follow-up of cases with and without surgical repair. Trans Am Acad Ophthalmol Otolaryngol 1971; 75: 802-812.

6 Koornneef L. Orbital septa: anatomy and function. Ophthalmology 1979; 86: 876-878.

7 Harris GJ, Garcia GH, Logani SC, Murphy ML. Correlation of preoperative computed tomography and postoperative ocular motility in orbital blow-out fractures. Ophthal Plast Reconstr Surg 2000; 16: 179-187.

8 Feibel RM, Roper-Hall G. Evaluation of the field of binocular single vision in incomitant strabismus. Am J Ophthalmol 1974; 78: 800-805.

9 Jordan DR, Allen LH, White J, Harvey J, Pashby R, Esmaeli B. Intervention within days for some orbital floor fractures: the white-eyed blowout. Ophthal Plast Reconstr Surg 1998; 14: 379-390.

10 Shorr N, Baylis HI, Goldberg RA, Perry JD. Transcaruncular approach to the medial orbit and orbital apex. Ophthalmology 2000; 107: 1459-1463. 